Document downloaded from:

http://hdl.handle.net/10251/52734

This paper must be cited as:

Benítez López, J. (2011). A projective invariant generalization of the de Casteljau algorithm. Computer-Aided Design. 43(1):3-11. doi:10.1016/j.cad.2010.09.005.

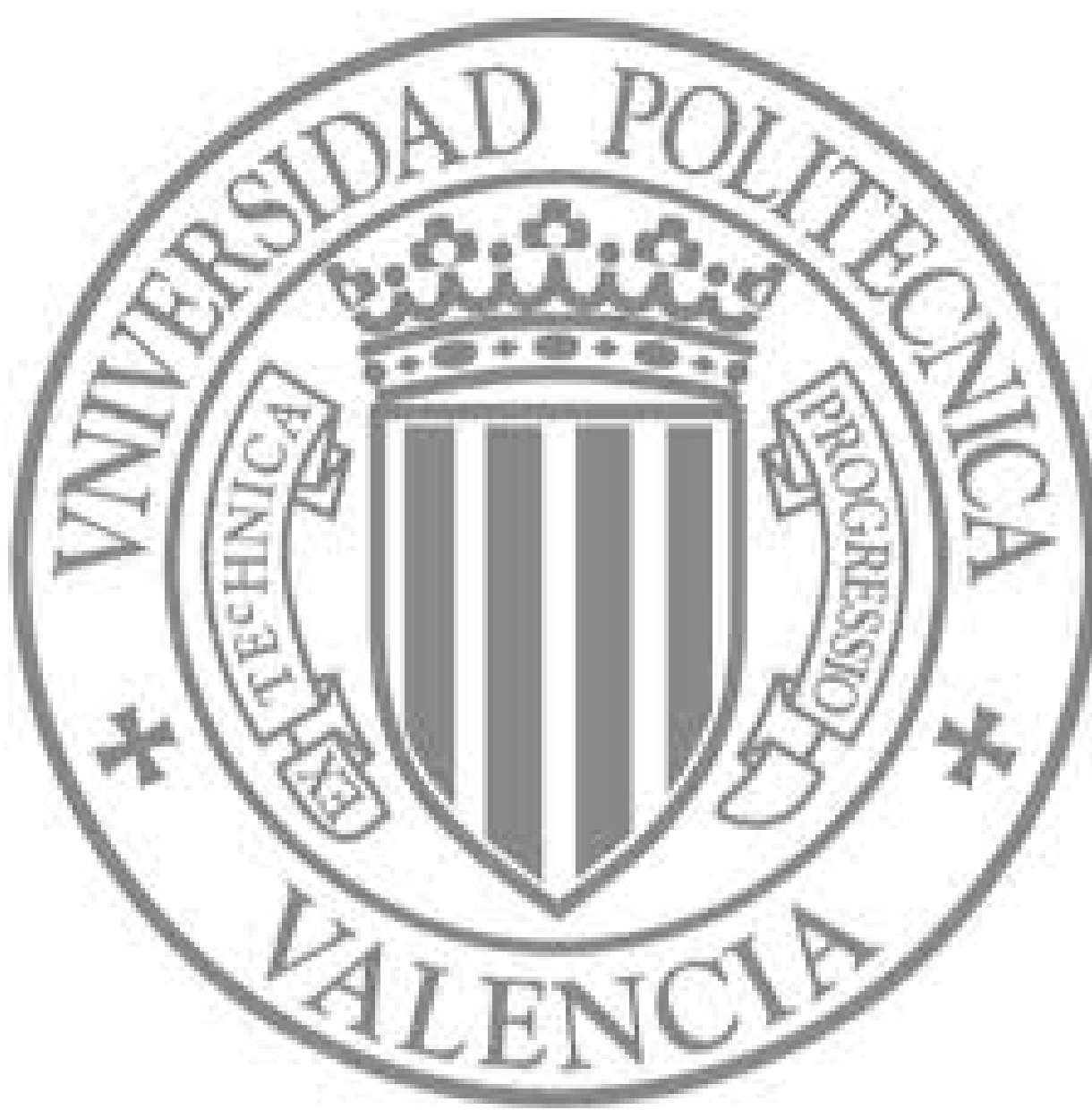

The final publication is available at

http://dx.doi.org/10.1016/j.cad.2010.09.005

Copyright Elsevier 


\title{
A projective invariant generalization of the de Casteljau algorithm
}

\author{
J. Benítez \\ Departamento de Matemática Aplicada. \\ Universidad Politécnica de Valencia. \\ Camino de Vera S/N. 46022. Valencia. Spain \\ jbenitez@mat.upv.es
}

\begin{abstract}
A projective invariant generalization of the de Casteljau algorithm is described by using the cross ratio and an auxiliary line. We describe the implicit form of the section conics obtained by the algorithm proposed in this paper. Finally, we show how to construct specific conic sections using this approach.
\end{abstract}

Keywords: De Casteljau algorithm; Projective invariance; Cross ratio; Rational Bézier curves.

\section{Introduction, background and notations}

It is well-known that the de Casteljau algorithm is affine invariant; but it is not projective invariant. Moreover, we can not design circles by using the de Casteljau algorithm (see for a simple proof [7, p. 25]). These two handicaps are overcome by using the rational Bézier curves, whose definition is the following (see, for example, [4] or [7] for a deeper study): A rational Bézier curve with control points $\mathbf{b}_{0}, \ldots, \mathbf{b}_{n} \in \mathbb{R}^{2}$ and weights $w_{0}, \ldots, w_{n} \in \mathbb{R}$ is given by

$$
\mathbf{r}(t)=\frac{\sum_{i=0}^{n} w_{i} \mathbf{b}_{i} B_{i}^{n}(t)}{\sum_{i=0}^{n} w_{i} B_{i}^{n}(t)}, \quad t \in[0,1],
$$


where $B_{i}^{n}(t)=\left(\begin{array}{c}n \\ i\end{array}\right)(1-t)^{n-i} t^{i}$ are the Bernstein polynomials. I.e., the curve $\mathbf{r}(t)$ is the central projection of the Bézier curve in $\mathbb{R}^{3}$ with control points $\left(w_{0} \mathbf{b}_{0}, w_{0}\right), \ldots,\left(w_{n} \mathbf{b}_{n}, w_{n}\right)$ (see [3] for this interpretation of the rational Bézier curves).

In this paper, a purely geometric construction is used to derive this class of curves by using concepts from projective geometry. Below we establish the basic ideas and notations used throughout this paper. The interested reader is referred to [1], [2], and [5] for a study of the properties of the real projective plane and their uses to computer graphics. All vectors are considered column vectors and for a given matrix $\mathbf{A}$, we shall denote by $\mathbf{A}^{\mathrm{T}}$ the transpose of $\mathbf{A}$.

We define a projective point as a Euclidian line in $\mathbb{R}^{3}$ that passes through the origin. The real projective plane $\mathbb{P}^{2}$ is the set of all projective points. If $P$ is a projective point, then there exists $\mathbf{v} \in \mathbb{R}^{3} \backslash\{\mathbf{0}\}$ such that $P$ is the line in $\mathbb{R}^{3}$ that passes through $\mathbf{0}$ and $\mathbf{v}$. Hence, we can define $\pi: \mathbb{R}^{3} \backslash\{\mathbf{0}\} \rightarrow \mathbb{P}^{2}$ as follows: $\pi(\mathbf{v})$ is the projective point that passes through $\mathbf{0}$ and $\mathbf{v}$. Observe that $\pi\left(\mathbf{v}_{1}\right)=\pi\left(\mathbf{v}_{2}\right)$ if and only if there exists a nonzero real number $\lambda$ such that $\mathbf{v}_{1}=\lambda \mathbf{v}_{2}$.

A projective line is a plane in $\mathbb{R}^{3}$ that passes through the origin and the set of all projectives lines will be denoted by $\Lambda\left(\mathbb{P}^{2}\right)$. We can define $\pi^{*}: \mathbb{R}^{3} \backslash\{\mathbf{0}\} \rightarrow \Lambda\left(\mathbb{P}^{2}\right)$ as follows: $\pi^{*}(\mathbf{w})$ is the projective line with equation $\mathbf{w}^{\mathrm{T}} \mathbf{x}=0$. Again one has that $\pi^{*}\left(\mathbf{w}_{1}\right)=\pi^{*}\left(\mathbf{w}_{2}\right)$ if and only if there exists a nonzero real number $\lambda$ such that $\mathbf{w}_{1}=\lambda \mathbf{w}_{2}$.

If $P_{1}$ and $P_{2}$ are two distinct projective points, then there exists a unique projective line through $P_{1}$ and $P_{2}$, such line shall be denoted by $\mathcal{L}\left(P_{1}, P_{2}\right)$. It is easy to see that the projective point $R$ lies on $\mathcal{L}(P, Q)$ if and only if there exist $\alpha, \beta \in \mathbb{R}$ not all zero such that $\mathbf{u}=\alpha \mathbf{v}+\beta \mathbf{w}$, where $\pi(\mathbf{u})=R$, $\pi(\mathbf{v})=P$, and $\pi(\mathbf{w})=Q$. If $r$ and $s$ are two distinct projective lines, then there is a unique projective point $P$ such that $\{P\}=r \cap s$.

The cross ratio is preserved under all projective transformations. This quantity is defined as follows: let four projective points $P_{1}, P_{2}, P_{3}, P_{4}$ be collinear. Thus, we can write $\mathbf{v}_{3}=\alpha \mathbf{v}_{1}+\beta \mathbf{v}_{2}$ and $\mathbf{v}_{4}=\gamma \mathbf{v}_{1}+\delta \mathbf{v}_{2}$ for some nonzero vectors $\mathbf{v}_{1}, \ldots, \mathbf{v}_{4}$ with $\pi\left(\mathbf{v}_{i}\right)=P_{i}$ for $i=1, \ldots, 4$. The cross ratio of $P_{1}, P_{2}, P_{3}, P_{4}$ is

$$
\operatorname{cr}\left(P_{1}, P_{2}, P_{3}, P_{4}\right)=\frac{\beta \gamma}{\alpha \delta} .
$$

It can be proved (see for example [2]) that this definition is well done, i.e., it does not depend on the choice of the vectors $\mathbf{v}_{i}$ such that $P_{i}=\pi\left(\mathbf{v}_{i}\right)$.

The ideal line is the projective line whose equation is $z=0$. An affine 
point is a projective point that does not belong to the ideal line. If $\mathcal{A}\left(\mathbb{P}^{2}\right)$ is the set of all affine points, we can establish two bijective maps in the following way:

$$
\begin{aligned}
\mathcal{E}^{2} & \stackrel{i}{\rightarrow} \mathcal{A}\left(\mathbb{P}^{2}\right) & \mathcal{A}\left(\mathbb{P}^{2}\right) & \stackrel{j}{\rightarrow} \mathcal{E}^{2} \\
(x, y)^{\mathrm{T}} & \mapsto \pi(x, y, 1)^{\mathrm{T}} & \pi(x, y, z)^{\mathrm{T}} & \mapsto\left(\frac{x}{z}, \frac{y}{z}\right)^{\mathrm{T}}
\end{aligned}
$$

where $\mathcal{E}^{2}$ is the symbol used for the Euclidean plane. We can easily check that $j \circ i=I_{\mathcal{E}^{2}}$ and $i \circ j=I_{\mathcal{A}\left(\mathbb{P}^{2}\right)}$, where $I$ denotes the identity map. For more details of the role of the ideal plane in projective geometry we refer to [1] and [2].

\section{The algorithm}

First of all we describe the geometric form of the algorithm (see Figure 1).

Algorithm 2.1 Given: the projective points $P_{0}, P_{1}, \ldots, P_{n} \in \mathbb{P}^{2}$, the projective line $r \in \Lambda\left(\mathbb{P}^{2}\right)$ such that $P_{i} \notin r$ for all $i=0, \ldots, n$ and $u \in \mathbb{R} \backslash\{1\}$.

Set: $P_{i}^{0}(u):=P_{i}$ for $i=0,1, \ldots, n$.

Suppose that the points $P_{i}^{k}$ have been constructed for $k=0, \ldots, j$ and $i=0, \ldots, n-k$. Now, for each $i=0, \ldots, n-j-1$

a) If $P_{i}^{j}(u)=P_{i+1}^{j}(u)$, then $P_{i}^{j+1}(u)=P_{i}^{j}(u)$.

b) If $P_{i}^{j}(u) \neq P_{i+1}^{j}(u)$, then let $Q_{i}^{j}(u)$ be the intersection of the projective lines $\mathcal{L}\left(P_{i}^{j}(u), P_{i+1}^{j}(u)\right)$ and $r$. Let $P_{i}^{j+1}(u)$ be on the line $\mathcal{L}\left(P_{i}^{j}(u), P_{i+1}^{j}(u)\right)$ such that

$$
\operatorname{cr}\left(P_{i}^{j}(u), P_{i+1}^{j}(u), P_{i}^{j+1}(u), Q_{i}^{j}(u)\right)=u,
$$

for $j=0, \ldots, n-1$.

The projective point $P_{0}^{n}(u)$ is the point with parameter value $u$ in the curve $\alpha\left(P_{0}, \ldots, P_{n} ; r\right): I \rightarrow \mathbb{P}^{2}$, where $I$ is an interval of $\mathbb{R}$ with $1 \notin I$.

We must prove that the algorithm is well defined, because in case b), if $r=\mathcal{L}\left(P_{i}^{j}(u), P_{i+1}^{j}(u)\right)$, then the projective point $Q_{i}^{j}(u)$ is not well defined. We need the following lemma:

Lemma 2.1 Let $\pi(\mathbf{u}), \pi(\mathbf{v}) \in \mathbb{P}^{2}$ and $\pi^{*}(\mathbf{w}) \in \Lambda\left(\mathbb{P}^{2}\right)$ such that $\pi(\mathbf{u}) \neq \pi(\mathbf{v})$ and $\pi(\mathbf{u}), \pi(\mathbf{v}) \notin \pi^{*}(\mathbf{w})$. Let $R=\mathcal{L}(\pi(\mathbf{u}), \pi(\mathbf{v})) \cap \pi^{*}(\mathbf{w})$. Then 


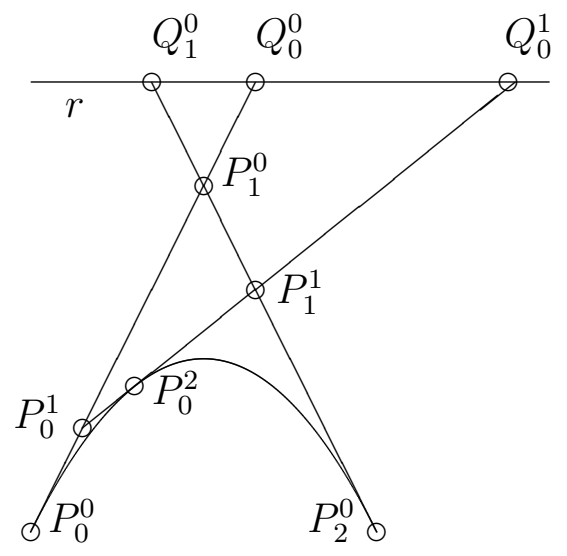

Figure 1: The geometric form of the algorithm.

a) $R=\pi\left[\left(\mathbf{w}^{\mathrm{T}} \mathbf{u}\right) \mathbf{v}-\left(\mathbf{w}^{\mathrm{T}} \mathbf{v}\right) \mathbf{u}\right]$.

b) If $S \in \mathbb{P}^{2}$ satisfies $\operatorname{cr}(\pi(\mathbf{u}), \pi(\mathbf{v}), S, R)=u$, then

$$
S=\pi\left[-\left(\mathbf{w}^{\mathrm{T}} \mathbf{u}\right) \mathbf{v}+u\left(\mathbf{w}^{\mathrm{T}} \mathbf{v}\right) \mathbf{u}\right] .
$$

Moreover, if $u \neq 1$, then $S \notin \pi^{*}(\mathbf{w})$.

Proof: a) It is easy to see that $\pi\left[\left(\mathbf{w}^{\mathrm{T}} \mathbf{u}\right) \mathbf{v}-\left(\mathbf{w}^{\mathrm{T}} \mathbf{v}\right) \mathbf{u}\right] \in \mathcal{L}(\pi(\mathbf{u}), \pi(\mathbf{v}))$ because $\left(\mathbf{w}^{\mathrm{T}} \mathbf{u}\right) \mathbf{v}-\left(\mathbf{w}^{\mathrm{T}} \mathbf{v}\right) \mathbf{u}$ is a linear combination of $\{\mathbf{u}, \mathbf{v}\}$. Moreover, $\pi\left[\left(\mathbf{w}^{\mathrm{T}} \mathbf{u}\right) \mathbf{v}-\left(\mathbf{w}^{\mathrm{T}} \mathbf{v}\right) \mathbf{u}\right] \in \pi^{*}(\mathbf{w})$ because $\mathbf{w}^{\mathrm{T}}\left[\left(\mathbf{w}^{\mathrm{T}} \mathbf{u}\right) \mathbf{v}-\left(\mathbf{w}^{\mathrm{T}} \mathbf{v}\right) \mathbf{u}\right]=0$.

b) In view of the previous item a) and the definition of cross ratio, it is clear that $S=\pi\left[-\left(\mathbf{w}^{\mathrm{T}} \mathbf{u}\right) \mathbf{v}+u\left(\mathbf{w}^{\mathrm{T}} \mathbf{v}\right) \mathbf{u}\right]$. Moreover, if $u \neq 1$,

$$
\begin{aligned}
\mathbf{w}^{\mathrm{T}}\left[-\left(\mathbf{w}^{\mathrm{T}} \mathbf{u}\right) \mathbf{v}+u\left(\mathbf{w}^{\mathrm{T}} \mathbf{v}\right) \mathbf{u}\right] & =-\left(\mathbf{w}^{\mathrm{T}} \mathbf{u}\right)\left(\mathbf{w}^{\mathrm{T}} \mathbf{v}\right)+u\left(\mathbf{w}^{\mathrm{T}} \mathbf{v}\right)\left(\mathbf{w}^{\mathrm{T}} \mathbf{u}\right) \\
& =(u-1)\left(\mathbf{w}^{\mathrm{T}} \mathbf{v}\right)\left(\mathbf{w}^{\mathrm{T}} \mathbf{u}\right) \neq 0 .
\end{aligned}
$$

In other words, $S \notin \pi^{*}(\mathbf{w})$.

REMARK 2.1 Clearly, by the item b) of the latter lemma, all projective lines $\mathcal{L}\left(P_{i}^{j}(u), P_{i+1}^{j}(u)\right)$ are different to $r$ provided that $u \neq 1$. Moreover, we obtain that $P_{i}^{j}(u) \notin r$ for all $i, j$ and $u \neq 1$.

Now, let us write the algorithm in algebraic form. We shall drop the parameter $u$ for the sake of simplicity. Let $\mathbf{v}_{i}^{j}, \mathbf{w} \in \mathbb{R}^{3}$ such that $\pi\left(\mathbf{v}_{i}^{j}\right)=P_{i}^{j}$ and $\pi^{*}(\mathbf{w})=r$. From Lemma 2.1, we get

$$
P_{i}^{j+1}=\pi\left[-\left(\mathbf{w}^{\mathrm{T}} \mathbf{v}_{i+1}^{j}\right) \mathbf{v}_{i}^{j}+u\left(\mathbf{w}^{\mathrm{T}} \mathbf{v}_{i}^{j}\right) \mathbf{v}_{i+1}^{j}\right] .
$$


Making $u=t /(t-1)$ we get

$$
P_{i}^{j+1}=\pi\left[(1-t)\left(\mathbf{w}^{\mathrm{T}} \mathbf{v}_{i+1}^{j}\right) \mathbf{v}_{i}^{j}+t\left(\mathbf{w}^{\mathrm{T}} \mathbf{v}_{i}^{j}\right) \mathbf{v}_{i+1}^{j}\right] .
$$

Thus, we can establish the algorithm in algebraic-projective form:

Algorithm 2.2 Given: the projective points $P_{0}, P_{1}, \ldots, P_{n} \in \mathbb{P}^{2}$, the projective line $r \in \Lambda\left(\mathbb{P}^{2}\right)$ such that $P_{i} \notin r$ for all $i=0, \ldots, n$ and $t \in \mathbb{R}$.

Set: $\mathbf{w} \in \mathbb{R}^{3}$ with $\pi^{*}(\mathbf{w})=r$ and set $P_{i}^{0}(t):=P_{i}$ for $i=0,1, \ldots, n$.

Suppose that the points $P_{i}^{k}(t)$ have been constructed and let $\mathbf{v}_{i}^{k}(t) \in \mathbb{R}^{3}$ with $\pi\left(\mathbf{v}_{i}^{k}(t)\right)=P_{i}^{k}(t)$ for $k=0, \ldots, j$ and $i=0, \ldots, n-k$. Now, for each $i=0, \ldots, n-j-1$, set

$$
P_{i}^{j+1}(t)=\pi\left[(1-t)\left(\mathbf{w}^{\mathrm{T}} \mathbf{v}_{i+1}^{j}(t)\right) \mathbf{v}_{i}^{j}(t)+t\left(\mathbf{w}^{\mathrm{T}} \mathbf{v}_{i}^{j}(t)\right) \mathbf{v}_{i+1}^{j}(t)\right],
$$

for $j=0, \ldots, n-1$.

Then the projective point $P_{0}^{n}(t)$ is the point with parameter value $t$ in the curve $\alpha\left(P_{0}, \ldots, P_{n} ; r\right): \mathbb{R} \rightarrow \mathbb{P}^{2}$.

In Figure 2 we can see the construction of a cubic in the affine plane by using the proposed algorithm. The affine points are calculated by means of the mapping $j$ defined in (2).

It is interesting to establish the algorithm by using vectors of $\mathbb{R}^{3}$. In order to simplify the equation (3) we observe that if $\mathbf{v}_{1}, \mathbf{v}_{2}$, and $\mathbf{w}$ are arbitrary vectors of $\mathbb{R}^{3}$ and if we set $\mathbf{v}=(1-t)\left(\mathbf{w}^{\mathrm{T}} \mathbf{v}_{2}\right) \mathbf{v}_{1}+t\left(\mathbf{w}^{\mathrm{T}} \mathbf{v}_{1}\right) \mathbf{v}_{2}$, being $t \in \mathbb{R}$, then $\mathbf{w}^{\mathrm{T}} \mathbf{v}=\left(\mathbf{w}^{\mathrm{T}} \mathbf{v}_{1}\right)\left(\mathbf{w}^{\mathrm{T}} \mathbf{v}_{2}\right)$.

Algorithm 2.3 Given: $\mathbf{v}_{0}, \mathbf{v}_{1}, \ldots, \mathbf{v}_{n}, \mathbf{w} \in \mathbb{R}^{3}$, such that $\mathbf{w}^{\mathrm{T}} \mathbf{v}_{i} \neq 0$ for all $i=0, \ldots, n$ and $t \in \mathbb{R}$.

Set: $\mathbf{v}_{i}^{0}(t)=\mathbf{v}_{i} /\left(\mathbf{w}^{\mathrm{T}} \mathbf{v}_{i}\right)$ for $i=0,1, \ldots, n$.

Suppose that the vectors $\mathbf{v}_{i}^{k}(t)$ have been constructed for $k=0, \ldots, j$ and $i=0, \ldots, n-k$. Now, for each $i=0, \ldots, n-j-1$, set

$$
\mathbf{v}_{i}^{j+1}(t):=(1-t) \mathbf{v}_{i}^{j}(t)+t \mathbf{v}_{i+1}^{j}(t)
$$

for $j=0, \ldots, n-1$ and $i=0,1, \ldots, n-j-1$.

Then $\mathbf{v}_{0}^{n}(t)$ is the vector with parameter $t$ in the curve $\beta\left(\mathbf{v}_{0}, \ldots, \mathbf{v} ; \mathbf{w}\right)$ : $\mathbb{R} \rightarrow \mathbb{R}^{3}$.

This algorithm allows us to infer two geometric properties: 


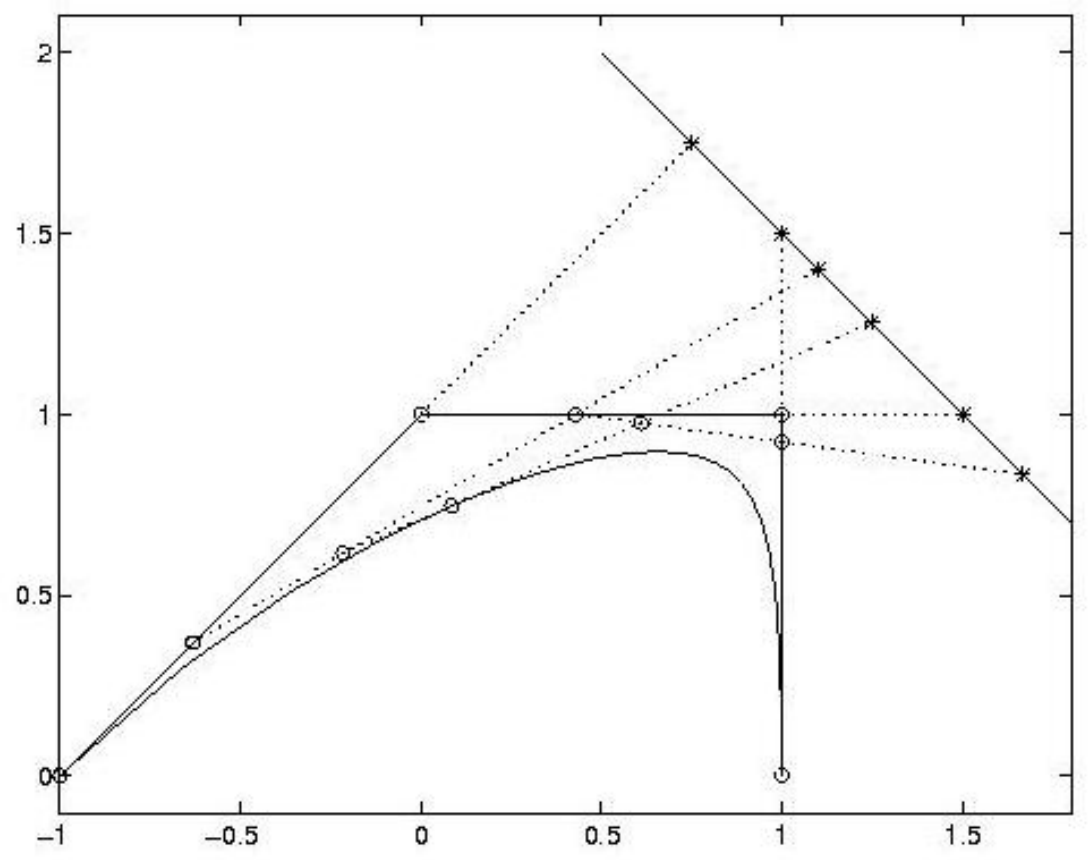

Figure 2: Construction of a cubic. The points $P_{i}^{j}(0.2)$ have been marked with "o". The points $Q_{i}^{j}(0.2)$ have been marked with "*”. In this figure, we have taken the following points: $P_{0}=i(-1,0)^{\mathrm{T}}, P_{1}=i(0,1)^{\mathrm{T}}, P_{2}=i(1,1)^{\mathrm{T}}$, $P_{3}=i(1,0)^{\mathrm{T}}$, and the line $\pi^{*}(1,1,-2.5)^{\mathrm{T}}$ (i.e., the image by $i$ of the affine line $x+y-2.5=0)$.

Projective invariance: Because the algorithm is concerned exclusively with projective properties it is evident that this algorithm is invariant under projective maps.

Duality: It is well known the duality principle in projective geometry: For any projective result established using points and lines, a symmetrical result holds if we interchange the roles of lines and points. So, we can easily establish the dual of the algorithm, recalling that the output is a set of projective lines (in the case of a conic section, we obtain the family of tangent lines to this curve). 
It is interesting to compare this algorithm with the introduced by Farin in [3]: In order to construct the rational Bézier curve (1), set

$$
\left.\begin{array}{c}
\mathbf{b}_{i}^{j+1}(t):=(1-t) \frac{w_{i}^{j}(t)}{w_{i}^{j+1}(t)} \mathbf{b}_{i}^{j}(t)+t \frac{w_{i+1}^{j}(t)}{w_{i}^{j+1}(t)} \mathbf{b}_{i+1}^{j}(t), \\
w_{i}^{j+1}(t):=(1-t) w_{i}^{j}(t)+t w_{i+1}^{j}(t) .
\end{array}\right\}
$$

The geometric interpretation of (5) is the following: let us define the points $\mathbf{q}_{i}^{j}(t)$ (called weight points or Farin points) located on the straight lines joining $\mathbf{b}_{i}^{j}(t)$ and $\mathbf{b}_{i+1}^{j}(t)$ such that

$$
\operatorname{ratio}\left(\mathbf{b}_{i}^{j}(t), \mathbf{q}_{i}^{j}(t), \mathbf{b}_{i+1}^{j}(t)\right)=\frac{w_{i+1}^{j}(t)}{w_{i}^{j}(t)}
$$

Then

$$
\operatorname{cr}\left(\mathbf{b}_{i}^{j}(t), \mathbf{q}_{i}^{j}(t), \mathbf{b}_{i}^{j+1}(t), \mathbf{b}_{i+1}^{j}(t)\right)=\frac{1-t}{t}
$$

for all $i, j$.

The algorithm 2.1 is different than the proposed in [3] because one of the inputs of the algorithm 2.1 is the auxiliary projective line $r$. An appropriate choice of the line $r$ gives more geometric insight as we shall show later. Moreover, as we will see, for $n=2$, this projective line will be tangent to the curve.

For $j=0$, the weight points

$$
\mathbf{q}_{i}=\mathbf{q}_{i}^{0}=\frac{w_{i} \mathbf{b}_{i}+w_{i+1} \mathbf{b}_{i+1}}{w_{i}+w_{i+1}}
$$

are directly related to the weights: given the weights, we can find the weight points and viceversa. Although, the weight points have resemblance with the points $Q_{i}^{j}$ defined in the Algorithm 2.1, they are different. This fact is easily seen in the Figure 3: the weight points are not collinear, but in the Algorithm 2.1, the points $Q_{i}^{j}$ are always collinear.

Let us see that the Algorithm 2.1 generalizes to the de Casteljau algorithm: Let $\mathbf{b}_{0}, \ldots, \mathbf{b}_{n}$ be points in $\mathcal{E}^{2}$. Let us apply the Algorithm 2.1 to the projective points $P_{0}=i\left(\mathbf{b}_{0}\right), \ldots, P_{n}=i\left(\mathbf{b}_{n}\right)$ and to the ideal line $r=\pi^{*}(\mathbf{w})$ with $\mathbf{w}=(0,0,1)^{\mathrm{T}}$. In view of the Remark 2.1 , we obtain that the projective 


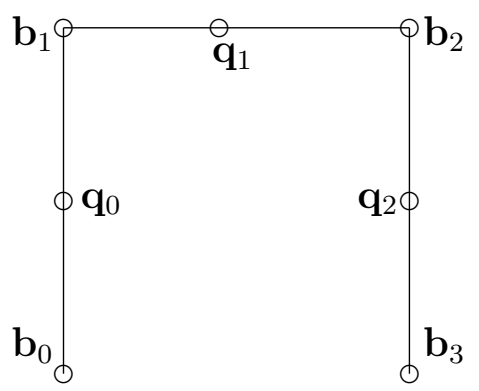

Figure 3: If we take $w_{0}=w_{1}=w_{2}=1$, then the weight points are the midpoints of $\overline{\mathbf{b}_{0} \mathbf{b}_{1}}, \overline{\mathbf{b}_{1} \mathbf{b}_{2}}$, and $\overline{\mathbf{b}_{2} \mathbf{b}_{3}}$.

points $P_{i}^{j}(t)$ are affine for all $i, j$, and $t \in \mathbb{R}$. Now, set $\mathbf{b}_{i}^{j}(t):=j\left(P_{i}^{j}(t)\right)$. It is clear that

$$
\mathbf{w}^{\mathrm{T}}\left(\begin{array}{c}
\mathbf{b}_{i}^{j}(t) \\
1
\end{array}\right)=1
$$

for all $i, j$, and $t \in \mathbb{R}$. Hence, by applying (3) and (6), one has

$$
\begin{aligned}
P_{i}^{j+1}(t) & =\pi\left[(1-t)\left(\begin{array}{c}
\mathbf{b}_{i}^{j}(t) \\
1
\end{array}\right)+t\left(\begin{array}{c}
\mathbf{b}_{i+1}^{j}(t) \\
1
\end{array}\right)\right] \\
& =\pi\left(\begin{array}{c}
(1-t) \mathbf{b}_{i}^{j}(t)+t \mathbf{b}_{i+1}^{j}(t) \\
1
\end{array}\right) .
\end{aligned}
$$

Therefore,

$$
\mathbf{b}_{i}^{j+1}(t)=j\left(P_{i}^{j+1}(t)\right)=(1-t) \mathbf{b}_{i}^{j}(t)+t \mathbf{b}_{i+1}^{j}(t) .
$$

This last equation is the main step in the de Casteljau's algorithm.

\section{Closed form}

Let $P_{0}=\pi\left(\mathbf{v}_{0}\right), P_{1}=\pi\left(\mathbf{v}_{1}\right), P_{2}=\pi\left(\mathbf{v}_{2}\right)$ be three projective points, and $r=\pi^{*}(\mathbf{w})$ a projective line such that $P_{i} \notin r$. Since $\mathbf{w}^{\mathrm{T}} \mathbf{v}_{i} \neq 0$, we can choose $\mathbf{v}_{i}$ such that $\mathbf{w}^{\mathrm{T}} \mathbf{v}_{i}=1$. By (4) we get that for $i=0,1$,

$$
\mathbf{v}_{i}^{1}(t)=(1-t) \mathbf{v}_{i}+t \mathbf{v}_{i+1} .
$$

And by (3), we have

$$
P_{0}^{2}(t)=\pi\left[(1-t) \mathbf{v}_{0}^{1}(t)+t \mathbf{v}_{1}^{1}(t)\right]
$$


Now, inserting (7) in (8) yields to

$$
P_{0}^{2}(t)=\pi\left[(1-t)^{2} \mathbf{v}_{0}+2 t(1-t) \mathbf{v}_{1}+t^{2} \mathbf{v}_{2}\right] .
$$

The following theorem is easily proved by induction:

THEOREM 3.1 The closed form of the projective curve $\alpha\left(P_{0}, \ldots, P_{n} ; r\right)$ is

$$
\alpha\left(P_{0}, \ldots, P_{n} ; r\right)(t)=\pi\left(\sum_{i=0}^{n} B_{i}^{n}(t) \mathbf{v}_{i}\right)
$$

where $B_{i}^{n}(t)=\left(\begin{array}{c}n \\ i\end{array}\right)(1-t)^{n-i} t^{i}$ are the Bernstein polynomials, $\pi\left(\mathbf{v}_{i}\right)=P_{i}$, and $\pi^{*}(\mathbf{w})=r$ with $\mathbf{w}^{\mathrm{T}} \mathbf{v}_{i}=1$ for $i=0, \ldots, n$.

REMARK 3.1 As an easy consequence of Theorem 3.1 we obtain that the vectors $\mathbf{v}_{1}-\mathbf{v}_{0}, \mathbf{v}_{n}-\mathbf{v}_{n-1}$ are tangent to the curve in $P_{0}, P_{n}$, respectively.

In the following, we shall see that the output of Algorithm 2.1 is a rational Bézier curve. Let $P_{0}, \ldots, P_{n}$ be points of $\mathbb{P}^{2}$ and $r$ a line in $\mathbb{P}^{2}$ such that $P_{i} \notin$ $r$. Let $\mathbf{v}_{i}=\left(x_{i}, y_{i}, z_{i}\right)^{\mathrm{T}}$ and $\mathbf{w}$ be nonzero vectors of $\mathbb{R}^{3}$ such that $\pi\left(\mathbf{v}_{i}\right)=P_{i}$ and $\pi^{*}(\mathbf{w})=r$. We shall obtain an expression for $j\left(\alpha\left(P_{0}, \ldots, P_{n} ; r\right)(t)\right)$, provided that $\alpha\left(P_{0}, \ldots, P_{n} ; r\right)(t)$ is affine. In order to apply Theorem 3.1, we define $\hat{\mathbf{v}}_{i}:=\mathbf{v}_{i} / \mathbf{w}^{\mathrm{T}} \mathbf{v}_{i}$, hence

$$
\begin{aligned}
\alpha\left(P_{0}, \ldots, P_{n} ; r\right)(t) & =\pi\left(\sum_{i=0}^{n} B_{i}^{n}(t) \hat{\mathbf{v}}\right)=\pi\left(\sum_{i=0}^{n} B_{i}^{n}(t) \frac{\mathbf{v}_{i}}{\mathbf{w}^{\mathrm{T}} \mathbf{v}_{i}}\right) \\
& =\pi\left(\sum_{i=0}^{n} \frac{B_{i}^{n}(t) x_{i}}{\mathbf{w}^{\mathrm{T}} \mathbf{v}_{i}}, \sum_{i=0}^{n} \frac{B_{i}^{n}(t) y_{i}}{\mathbf{w}^{\mathrm{T}} \mathbf{v}_{i}}, \sum_{i=0}^{n} \frac{B_{i}^{n}(t) z_{i}}{\mathbf{w}^{\mathrm{T}} \mathbf{v}_{i}}\right)^{\mathrm{T}}
\end{aligned}
$$

If we set

$$
w(t)=\sum_{i=0}^{n} \frac{B_{i}^{n}(t) z_{i}}{\mathbf{w}^{\mathrm{T}} \mathbf{v}_{i}}
$$

and we suppose that $w(t) \neq 0$ (i.e., $\alpha\left(P_{0}, \ldots, P_{1} ; r\right)(t)$ is an affine point), then

$$
j\left(\alpha\left(P_{0}, \ldots, P_{1} ; r\right)(t)\right)=\frac{1}{w(t)}\left(\sum_{i=0}^{n} \frac{B_{i}^{n}(t) x_{i}}{\mathbf{w}^{\mathrm{T}} \mathbf{v}_{i}}, \sum_{i=0}^{n} \frac{B_{i}^{n}(t) y_{i}}{\mathbf{w}^{\mathrm{T}} \mathbf{v}_{i}}\right) .
$$


Note that if $z_{i}=1$, then (11) is a rational Bézier curve with weights $\left(\mathbf{w}^{\mathrm{T}} \mathbf{v}_{i}\right)^{-1}$. Furthermore, if $r$ is not the ideal line then the equation of the line $r$ is $a x+b y+c z=0$ with $a^{2}+b^{2} \neq 0$. Thus

$$
\left|\mathbf{w}^{\mathrm{T}} \mathbf{v}_{i}\right|=\left|a x_{i}+b y_{i}+c\right|=\sqrt{a^{2}+b^{2}} \frac{\left|a x_{i}+b y_{i}+c\right|}{\sqrt{a^{2}+b^{2}}}=\sqrt{a^{2}+b^{2}} \mathrm{~d}\left(\mathbf{b}_{i}, j(r)\right),
$$

where $\mathbf{b}_{i}=\left(x_{i}, y_{i}\right)^{\mathrm{T}}$ and $\mathrm{d}\left(\mathbf{b}_{i}, j(r)\right)$ is the distance between the affine line $j(r)$ and the point $\mathbf{b}_{i}$. Thus, the lesser is $\mathrm{d}\left(\mathbf{b}_{i}, j(r)\right)$, the greater is the weight associated to the point $\mathbf{b}_{i}$. If we have an interactive algorithm, the designer should not be required to know any mathematics and the program should give the designer a intuitive handle which can be used in order to manage the output. Recall that the line $r$ is one of the inputs of the algorithm.

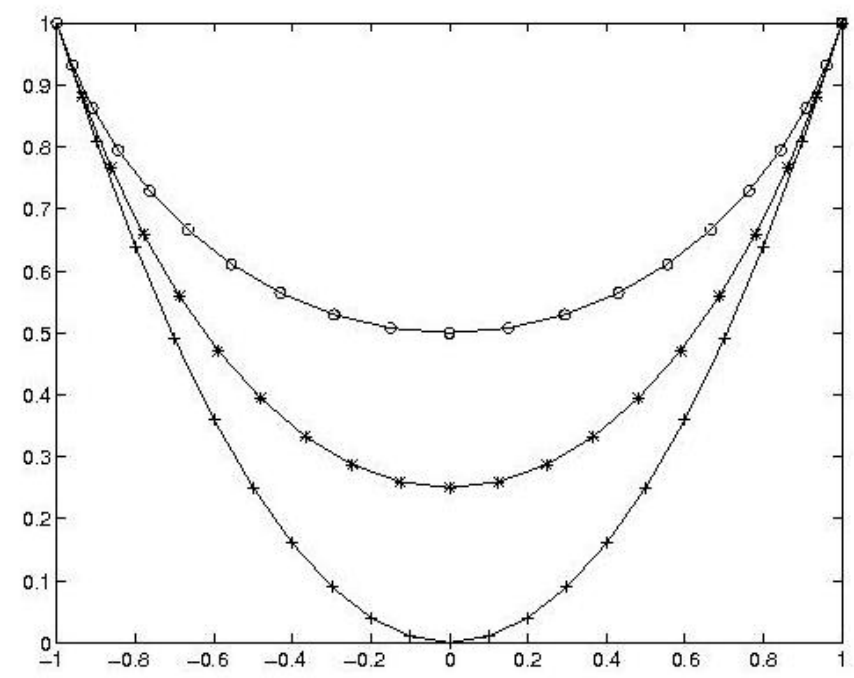

Figure 4: Three different curves designed with the Algorithm 2.1. The control points are $i(-1,1)^{\mathrm{T}}, i(0,-1)^{\mathrm{T}}, i(1,1)^{\mathrm{T}}$. The curve with "+" was depicted with the standard de Casteljau algorithm. The curves with "*" and "o" were depicted by using the affine lines $y=4$ and $y=2$ respectively.

In Figure 4 we can observe that if the line $r$ approaches to the ideal line, then the curve is closer to the parabola created by the de Casteljau algorithm. We can see in Figure 5 some other examples of the behaviour of the curve 
$j\left(\alpha\left(P_{0}, \ldots, P_{n} ; r\right)\right)$ when $r$ moves. In an intuitive way, the line $r$ acts as a magnet attracting to this curve. Thus, we can see in these examples how this auxiliary line $r$ permits designers to change the shape of the curve in an intuitive way.
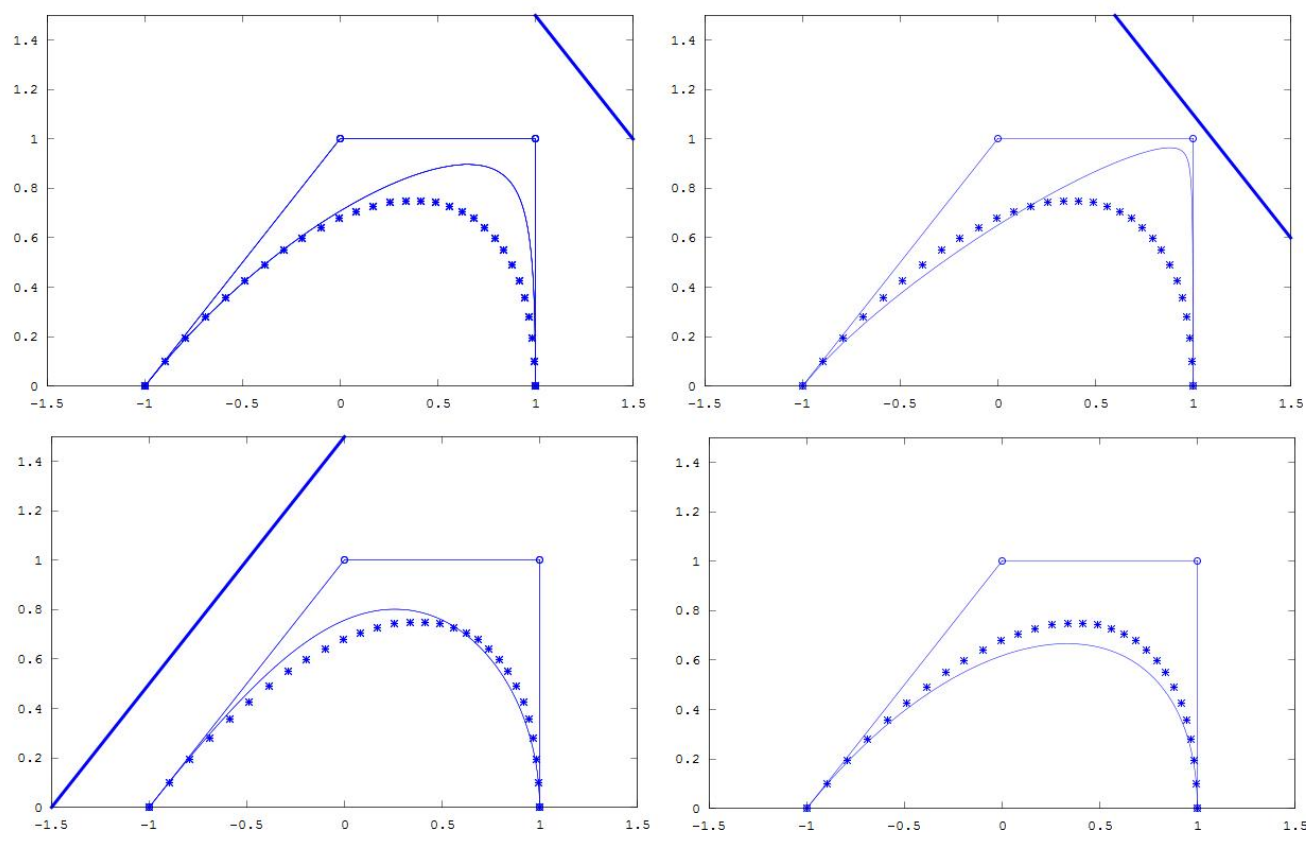

Figure 5: The line $r$ as an attractor. The control points are $i(-1,0)^{\mathrm{T}}, i(0,1)^{\mathrm{T}}$, $i(1,1)^{\mathrm{T}}$, and $i(1,0)^{\mathrm{T}}$. The curve whose points are marked with "**" is generated with the standard de Casteljau's Algorithm. The lines are: upper-left, $x+y=2.5$; upper-right, $x+y=2.1$; lower-left, $x-y=-1.5$; lower-right, $y=-2$ (this line is not drawn).

Under the assumptions of the last paragraph, note that all weights have the same sign if and only if all points $\mathbf{b}_{i}$ are in the same half-plane on one side of the line $r$.

Another advantage of the algorithm is that it permits to manage simultaneously affine points and "infinite control points": in equation (11), it was not assumed that $z_{i} \neq 0$. (see, for example, [4] for a different treatment).

We are going to prove in next result that any rational Bézier curve written 
as in (1), when $n=2$, is the output of the algorithm proposed in this paper.

TheOREM 3.2 Let $\mathbf{b}_{0}, \mathbf{b}_{1}, \mathbf{b}_{2} \in \mathbb{R}^{2}$ be non collinear points and $w_{0}, w_{1}, w_{n}>$ 0 . If $\mathbf{r}$ is written as in (1), then there exists $P_{0}, P_{1}, P_{2} \in \mathbb{P}^{2}$ and $r \in \Lambda\left(\mathbb{P}^{2}\right)$ such that $\mathbf{r}=j\left(\alpha\left(P_{0}, P_{1}, P_{2} ; r\right)\right)$.

Proof: Let us define $\mathbf{v}_{i}=\left[\begin{array}{c}\mathbf{b}_{i} \\ 1\end{array}\right]$ for $i=0,1,2$. If we prove that there exists $\mathbf{w} \in \mathbb{R}^{3}$ such that

$$
\mathbf{w}^{\mathrm{T}} \mathbf{v}_{i}=w_{n}^{-1} \quad i=0,1,2,
$$

then (10) and (11) will prove this Theorem. But observe that (12) is equivalent to $\mathbf{w}^{\mathrm{T}}\left[\mathbf{v}_{0} \mathbf{v}_{1} \mathbf{v}_{2}\right]=\left[\begin{array}{lll}w_{0}^{-1} & w_{1}^{-1} & w_{2}^{-1}\end{array}\right]$. But matrix $\left[\begin{array}{lll}\mathbf{v}_{0} & \mathbf{v}_{1} & \mathbf{v}_{2}\end{array}\right]$ is nonsingular because the points $\mathbf{b}_{0}, \mathbf{b}_{1}$, and $\mathbf{b}_{2}$ are not collinear. Thus, if we define

$$
\mathbf{w}=\left[\begin{array}{l}
\mathbf{v}_{0}^{\mathrm{T}} \\
\mathbf{v}_{1}^{\mathrm{T}} \\
\mathbf{v}_{2}^{\mathrm{T}}
\end{array}\right]^{-1}\left[\begin{array}{l}
w_{0}^{-1} \\
w_{1}^{-1} \\
w_{2}^{-1}
\end{array}\right],
$$

then (12) holds. The Theorem is thus proved.

REMARK 3.2 By mimicking the proof of former Theorem 3.2, one gets the following stronger result (although more artificial): Let $\mathbf{b}_{0}, \ldots, \mathbf{b}_{n} \in \mathbb{R}^{2}$ and $w_{0}, \ldots, w_{n}>0$. If there exists $\mathbf{w} \in \mathbb{R}^{3}$ such that $\mathbf{w}^{\mathrm{T}} \mathbf{v}_{i}=w_{i}^{-1}$ for $i=$ $0, \ldots, n$, where $\mathbf{v}_{i}$ are defined by $\mathbf{v}_{i}^{\mathrm{T}}=\left[\mathbf{b}_{n}^{T} 1\right]$, then

$$
\mathbf{r}=j\left(\alpha\left(\pi\left(\mathbf{v}_{1}\right), \ldots, \pi\left(\mathbf{v}_{i}\right) ; \pi^{*}(\mathbf{w})\right)\right) .
$$

Now, let us see that for $n=2$, the algorithm produces a projective conic. Moreover, we shall obtain the implicit form of this conic, which has an important application: check if a given point lies on the conic. Recall that a projective conic may be written as

$$
\mathcal{C}=\left\{\pi(\mathbf{x}) \in \mathbb{P}^{2}: \mathbf{x}^{\mathrm{T}} \mathbf{B} \mathbf{x}=0\right\},
$$

where $\mathbf{x} \in \mathbb{R}^{3}$ and $\mathbf{B}$ is a symmetric $3 \times 3$ matrix (see, for example, [1]).

In order to write the curve described in (9) in the form (13), we need the following simple observation: if $\mathbf{r}: \mathbb{R} \rightarrow \mathbb{R}^{3}$ is given by

$$
\mathbf{r}(t)=\left((1-t)^{2}, 2 t(1-t), t^{2}\right)^{\mathrm{T}}=\sum_{i=0}^{2} B_{i}^{2}(t) \mathbf{e}_{i+1},
$$


where $\left\{\mathbf{e}_{1}, \mathbf{e}_{2}, \mathbf{e}_{3}\right\}$ is the standard basis of $\mathbb{R}^{3}$, then

$$
\mathbf{r}(t)^{\mathrm{T}} \mathbf{J r}(t)=0
$$

with

$$
\mathbf{J}=\left[\begin{array}{ccc}
0 & 0 & 2 \\
0 & -1 & 0 \\
2 & 0 & 0
\end{array}\right]
$$

If we set $\mathbf{A}:=\left[\mathbf{v}_{0}, \mathbf{v}_{1}, \mathbf{v}_{2}\right]^{-1}$ (if $\left\{\mathbf{v}_{0}, \mathbf{v}_{1}, \mathbf{v}_{2}\right\}$ were not independent then the points $P_{0}, P_{1}, P_{2}$ would be collinear) then $\mathbf{A} \mathbf{v}_{i}=\mathbf{e}_{i+1}$ for $i=0,1,2$. Denoting $\mathbf{s}(t):=\sum_{i=0}^{2} B_{i}^{2}(t) \mathbf{v}_{i}$ we get

$$
\mathbf{r}(t)=\sum_{i=0}^{2} B_{i}^{2}(t) \mathbf{e}_{i+1}=\sum_{i=0}^{2} B_{i}^{2}(t) \mathbf{A} \mathbf{v}_{i}=\mathbf{A} \sum_{i=0}^{2} B_{i}^{2}(t) \mathbf{v}_{i}=\mathbf{A} \mathbf{s}(t)
$$

By inserting this latter equation in (14) we get

$$
0=(\mathbf{A s}(t))^{\mathrm{T}} \mathbf{J}(\mathbf{A} \mathbf{s}(t))=\mathbf{s}(t)^{\mathrm{T}}\left(\mathbf{A}^{\mathrm{T}} \mathbf{J} \mathbf{A}\right) \mathbf{s}(t) .
$$

So, we have proved the following result.

TheOREM 3.3 The curve parametrized by $\alpha\left(P_{0}, P_{1}, P_{2} ; r\right)$ is a conic and satisfies the equation $\mathbf{x}^{\mathrm{T}}\left(\mathbf{A}^{\mathrm{T}} \mathbf{J} \mathbf{A}\right) \mathbf{x}=0$, where $\mathbf{J}$ is defined in (15), $\mathbf{A}=$ $\left[\mathbf{v}_{0}, \mathbf{v}_{1}, \mathbf{v}_{2}\right]^{-1}, \pi\left(\mathbf{v}_{i}\right)=P_{i}$, and $r=\pi^{*}(\mathbf{w})$ with $\mathbf{w}^{\mathrm{T}} \mathbf{v}_{i}=1$.

Note that $\mathbf{A}^{\mathrm{T}} \mathbf{J A}$ is a nonsingular matrix, which means that the conic is non degenerate. Since

$$
\begin{aligned}
\left(\mathbf{A}^{\mathrm{T}} \mathbf{J} \mathbf{A}\right)^{-1} & =\mathbf{A}^{-1} \mathbf{J}^{-1} \mathbf{A}^{-\mathrm{T}}=\left[\mathbf{v}_{0}, \mathbf{v}_{1}, \mathbf{v}_{2}\right]\left[\begin{array}{ccc}
0 & 0 & 1 / 2 \\
0 & -1 & 0 \\
1 / 2 & 0 & 0
\end{array}\right]\left[\begin{array}{c}
\mathbf{v}_{0}^{\mathrm{T}} \\
\mathbf{v}_{1}^{\mathrm{T}} \\
\mathbf{v}_{2}^{\mathrm{T}}
\end{array}\right] \\
& =\frac{1}{2}\left(\mathbf{v}_{2} \mathbf{v}_{0}^{\mathrm{T}}+\mathbf{v}_{0} \mathbf{v}_{2}^{\mathrm{T}}\right)-\mathbf{v}_{1} \mathbf{v}_{1}^{\mathrm{T}}
\end{aligned}
$$

we get

$$
\mathbf{A}^{\mathrm{T}} \mathbf{J} \mathbf{A}=\left(\frac{1}{2}\left(\mathbf{v}_{2} \mathbf{v}_{0}^{\mathrm{T}}+\mathbf{v}_{0} \mathbf{v}_{2}^{\mathrm{T}}\right)-\mathbf{v}_{1} \mathbf{v}_{1}^{\mathrm{T}}\right)^{-1},
$$

which is faster than to compute first $\mathbf{A}=\left[\mathbf{v}_{0}, \mathbf{v}_{1}, \mathbf{v}_{2}\right]^{-1}$ and after $\mathbf{A}^{\mathrm{T}} \mathbf{J} \mathbf{A}$. Equation (16) has an important consequence. Recall that for a projective conic in the form (13), if $\mathbf{B}$ is nonsingular, then the equation of the tangent lines to $\mathcal{C}$ is $\left\{\pi^{*}(\mathbf{w}): \mathbf{w}^{\mathrm{T}} \mathbf{B}^{-1} \mathbf{w}=0\right\}$. 
TheOREM 3.4 Under the hypothesis of Theorem 3.3, the line $r$ is tangent to the conic. Moreover, if $\mathbf{r}: \mathbb{R} \rightarrow \mathbb{P}^{2}$ is the parametrization of the conic depicted by the Algorithm 2.1 and there exists $\lim _{t \rightarrow \pm \infty} \mathbf{r}(t)=\mathbf{x}_{0}$, then $r$ is tangent at $\mathbf{x}_{0}$.

Proof: It is enough to check $\mathbf{w}^{\mathrm{T}}\left(\mathbf{A}^{\mathrm{T}} \mathbf{J} \mathbf{A}\right)^{-1} \mathbf{w}=0$, but, this is easy in view of the equation (16) and $\mathbf{w}^{\mathrm{T}} \mathbf{v}_{i}=\mathbf{v}_{i}^{\mathrm{T}} \mathbf{w}=1$ for $i=0,1,2$. The last affirmation follows from Remark 2.1.

Figure 7 explains Theorem 3.4. From now on, we will denote $\mathbf{r}(\infty)=$ $\lim _{t \rightarrow \pm \infty} \mathbf{r}(t)$ when this limit exists.

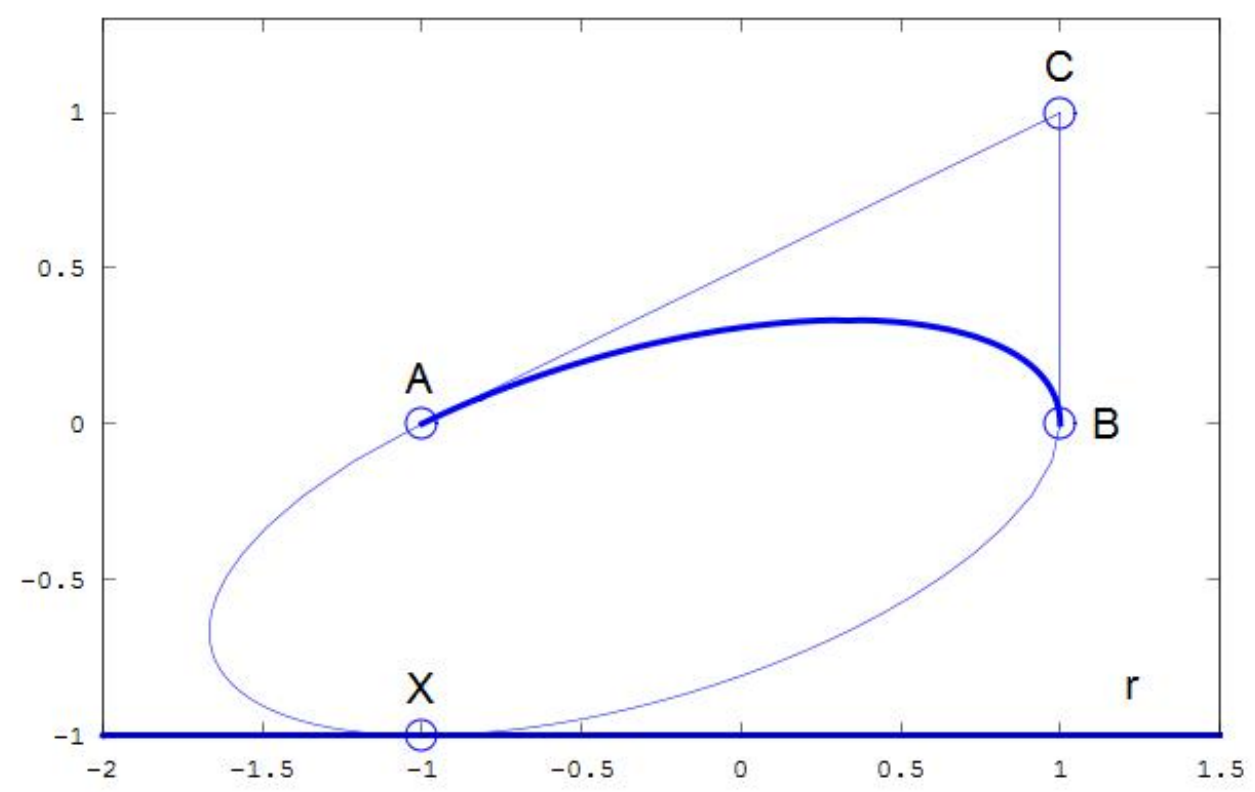

Figure 6: ***

We shall compare the implicit form of a conic given in Theorem 3.3 with the proposed in $[4$, p. 206] which we state here for the sake of readability: For a given conic

$$
\mathbf{r}(t)=\frac{\sum_{i=0}^{2} w_{i} B_{i}^{2}(t) \mathbf{b}_{i}}{\sum_{i=0}^{2} w_{i} B_{i}^{2}(t)},
$$

with $\mathbf{b}_{i}=\left(b_{i}^{x}, b_{i}^{y}\right)^{\mathrm{T}} \in \mathcal{E}^{2}$ and $w_{i} \in \mathbb{R}$. The point $\mathbf{c}=\left(c^{x}, c^{y}\right)^{\mathrm{T}} \in \mathcal{E}^{2}$ lies on 


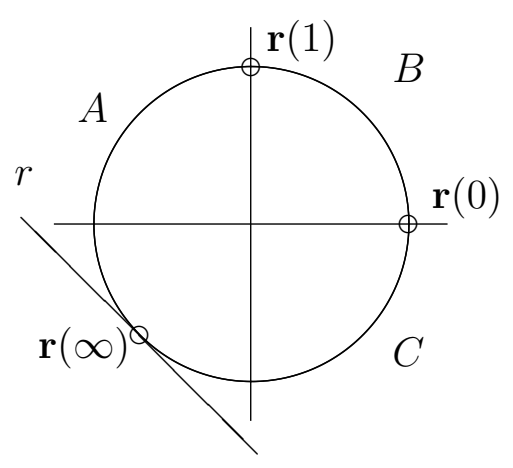

Figure 7: The unit circle has been partitioned in three disjoint arcs: $A, B$, and $C$, being $A=\{\mathbf{r}(t): 1<t\}, B=\{\mathbf{r}(t): 0 \leq t \leq 1\}$, and $C=\{\mathbf{r}(t): t<0\}$.

the conic (17) if and only if

$$
\frac{\tau_{1}^{2}}{\tau_{0} \tau_{2}}=4 \frac{w_{1}^{2}}{w_{0} w_{2}}
$$

where

$$
\tau_{0}=\frac{\left|\begin{array}{lll}
c^{x} & c^{y} & 1 \\
b_{1}^{x} & b_{1}^{y} & 1 \\
b_{2}^{x} & b_{2}^{y} & 1
\end{array}\right|}{\left|\begin{array}{lll}
b_{0}^{x} & b_{0}^{y} & 1 \\
b_{1}^{x} & b_{1}^{y} & 1 \\
b_{2}^{x} & b_{2}^{y} & 1
\end{array}\right|}, \quad \tau_{1}=\frac{\left|\begin{array}{ccc}
b_{0}^{x} & b_{0}^{y} & 1 \\
c^{x} & c^{y} & 1 \\
b_{2}^{x} & b_{2}^{y} & 1
\end{array}\right|}{\left|\begin{array}{ccc}
b_{0}^{x} & b_{0}^{y} & 1 \\
b_{1}^{x} & b_{1}^{y} & 1 \\
b_{2}^{x} & b_{2}^{y} & 1
\end{array}\right|}, \quad \tau_{0}=\frac{\left|\begin{array}{ccc}
b_{0}^{x} & b_{0}^{y} & 1 \\
b_{1}^{x} & b_{1}^{y} & 1 \\
c^{x} & c^{y} & 1
\end{array}\right|}{\left|\begin{array}{ccc}
b_{0}^{x} & b_{0}^{y} & 1 \\
b_{1}^{x} & b_{1}^{y} & 1 \\
b_{2}^{x} & b_{2}^{y} & 1
\end{array}\right|}
$$

are the barycentric coordinates of $\mathbf{c}$ respect to $\left\{\mathbf{b}_{0}, \mathbf{b}_{1}, \mathbf{b}_{2}\right\}$, i.e. $\mathbf{c}=\tau_{0} \mathbf{b}_{0}+$ $\tau_{1} \mathbf{b}_{1}+\tau_{2} \mathbf{b}_{2}$.

Although computationally these two approaches are equivalent, Theorem 3.3 is preferable by two features. $1^{\text {st }}$ : In (17) the control points $\mathbf{b}_{i}$ must be affine, while in Theorem 3.3 we do not need that the projective points $\pi\left(\mathbf{v}_{i}\right)$ are affine. $2^{\text {nd }}$ : In many standard textbooks of analytic geometry, a classification of a conic given in the form (13) can be found. Moreover, there are expressions for the center, tangent lines, asymptotes, ... for a given matrix in the form (13). 


\section{Examples}

We shall construct some specific conic sections using the approach presented in this paper:

Example 1: Given $\mathbf{c}, \mathbf{p}_{0}, \mathbf{p}_{1} \in \mathcal{E}^{2}$ with $\left\|\mathbf{p}_{0}-\mathbf{c}\right\|=\left\|\mathbf{p}_{1}-\mathbf{c}\right\|=\rho$, and $\mathbf{p}_{0}-\mathbf{c} \perp \mathbf{p}_{1}-\mathbf{c}$, draw the quadrant $\mathbf{p}_{0} \mathbf{p}_{1}$ of the circle centered at $\mathbf{c}$ and radius $\rho$ (see Figure 8 ).

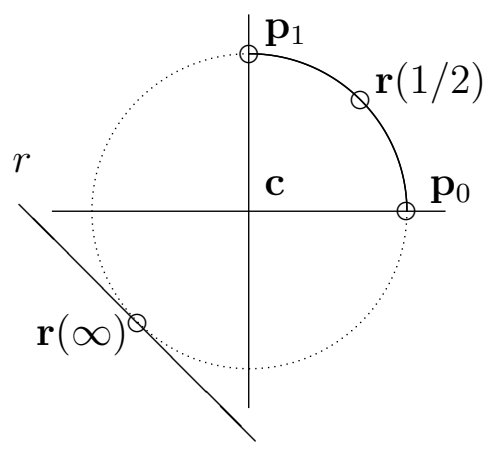

Figure 8: Construction of the quadrant of the unit circle.

One solution (presented in [7]) is the following: By the affine invariance of the rational Bézier curves we can suppose $\mathbf{p}_{0}=(1,0)^{\mathrm{T}}, \mathbf{p}_{1}=(0,1)^{\mathrm{T}}$, and $\mathbf{c}=(0,0)^{\mathrm{T}}$. Making $t=\tan (u / 2)$ in the trigonometric parametrization $\mathbf{r}(u)=(\cos u, \sin u)$ for $0 \leq u \leq \pi / 2$ we obtain

$$
\mathbf{r}(t)=\left(\frac{1-t^{2}}{1+t^{2}}, \frac{2 t}{1+t^{2}}\right)^{\mathrm{T}}, \quad 0 \leq t \leq 1
$$

This parametrization is in the form (17) in which

$$
\mathbf{b}_{0}=\left(\begin{array}{c}
1 \\
0
\end{array}\right), \mathbf{b}_{1}=\left(\begin{array}{l}
1 \\
1
\end{array}\right), \mathbf{b}_{0}=\left(\begin{array}{c}
0 \\
1
\end{array}\right), w_{0}=1, w_{1}=1, w_{2}=2 .
$$

This is not geometrically intuitive due to a lack of symmetry between the weights $w_{0}$ and $w_{2}$. Moreover, setting $t=1 / 2$ in (18) we obtain $\mathbf{r}(1 / 2)=$ $(0.6,0.8)^{\mathrm{T}}$, which is not the midpoint of the circular arc in the first quadrant. The point $\mathbf{r}(1 / 2)$ is closer to $\mathbf{r}(1)$ than $\mathbf{r}(0)$, which is explained by noticing 
that $\left\|\mathbf{r}^{\prime}(0)\right\|$ is twice $\left\|\mathbf{r}^{\prime}(1)\right\|$. In $[7$, Ch. 6] this asymmetry is solved by a reparametrization.

We shall obtain a parametrization of the first quadrant of the unit circle using the Algorithm 2.1 with more geometric flavour. Note that the auxiliary line $r$ will play an important role.

In order to apply the Algorithm 2.1, set $\mathbf{v}_{0}=(1,0,1)^{\mathrm{T}}$ and $\mathbf{v}_{2}=(0,1,1)^{\mathrm{T}}$. By Remark 3.1, we must define $\mathbf{v}_{1}=(1,1,1)^{\mathrm{T}}$. By Theorem 3.4, the line $r$ is tangent to the circle. By symmetry and because we want to draw the first quadrant, let $r$ be the line whose equation is $x+y=-\sqrt{2}$ (see Figure 8). Therefore, following the notation used throughout this paper, we set $\mathbf{w}=(1,1, \sqrt{2})^{\mathrm{T}}$. By applying (10) and (11) we obtain

$$
w(t)=\frac{(1-t)^{2}}{1+\sqrt{2}}+\frac{2 t(1-t)}{2+\sqrt{2}}+\frac{t^{2}}{1+\sqrt{2}}
$$

and

$$
\mathbf{r}(t)=\frac{1}{w(t)}\left(\frac{(1-t)^{2}}{1+\sqrt{2}}+\frac{2 t(1-t)}{2+\sqrt{2}}, \frac{2 t(1-t)}{2+\sqrt{2}}+\frac{t^{2}}{1+\sqrt{2}}\right)^{\mathrm{T}} .
$$

We can observe that $\mathbf{r}(1 / 2)=\left(\cos \frac{\pi}{4}, \sin \frac{\pi}{4}\right)^{\mathrm{T}}$ and $\mathbf{r}(\infty)=\left(-\cos \frac{\pi}{4},-\sin \frac{\pi}{4}\right)^{\mathrm{T}}$, as Figure 8 shows.

We can prove that the curve (19) is indeed part of the unit circle by simplifying $\|\mathbf{r}(t)\|$, but this approach is very tedious. A faster method is to apply Theorem 3.3. In order to use this theorem, we redefine

$$
\mathbf{v}_{0}=\frac{1}{1+\sqrt{2}}\left(\begin{array}{l}
1 \\
0 \\
1
\end{array}\right), \quad \mathbf{v}_{1}=\frac{1}{2+\sqrt{2}}\left(\begin{array}{l}
1 \\
1 \\
1
\end{array}\right), \quad \mathbf{v}_{2}=\frac{1}{1+\sqrt{2}}\left(\begin{array}{l}
0 \\
1 \\
1
\end{array}\right)
$$

with the purpose to have $\mathbf{w}^{\mathrm{T}} \mathbf{v}_{i}=1$. A very easy computation gives

$$
\left(\mathbf{A}^{\mathrm{T}} \mathbf{J} \mathbf{A}\right)^{-1}=\frac{1}{2}\left(\mathbf{v}_{2} \mathbf{v}_{0}^{\mathrm{T}}+\mathbf{v}_{0} \mathbf{v}_{2}^{\mathrm{T}}\right)-\mathbf{v}_{1} \mathbf{v}_{1}^{\mathrm{T}}=\left(\begin{array}{ccc}
\sqrt{2}-\frac{3}{2} & 0 & 0 \\
0 & \sqrt{2}-\frac{3}{2} & 0 \\
0 & 0 & -\sqrt{2}+\frac{3}{2}
\end{array}\right) .
$$

Therefore, matrix $\mathbf{A}^{\mathrm{T}} \mathbf{J} \mathbf{A}$ is a scalar multiple of $\left(\begin{array}{ccc}1 & 0 & 0 \\ 0 & 1 & 0 \\ 0 & 0 & -1\end{array}\right)$, which implies that the conic is the circle $x^{2}+y^{2}-1=0$. 
Example 2: If we want to draw the complementary of the arc depicted in the previous example, it is enough to set $\mathbf{v}_{0}=(1,0,1)^{\mathrm{T}}, \mathbf{v}=(1,1,1)^{\mathrm{T}}$, and $\mathbf{v}_{2}=(0,1,1)^{\mathrm{T}}$ (as in the Example 1 ) and consider the line $r$ with equation $x+y=\sqrt{2}$ (see Figure 9 ). Therefore, following the notation used throughout this paper, we set $\mathbf{w}=(1,1,-\sqrt{2})^{\mathrm{T}}$.

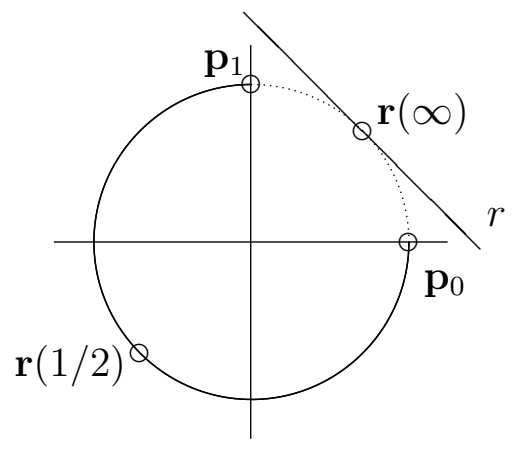

Figure 9: Construction of a circular arc with sweep angle equal to $3 \pi / 2$.

Example 3: Draw an arc of a circle whose amplitude is equal to $\theta \in] 0, \pi[$ (in the example 4 we will show how to construct a semicircle and in example 2 we saw how to draw an arc with sweep angle greater than $\pi$ ).

We will use the shorthand notation $c$ for $\cos \theta$ and $s$ for $\sin \theta$. As in the previous examples, we can suppose that the radius is 1 and the circle is centered at the origin. Set $\mathbf{p}_{0}=(1,0)^{\mathrm{T}}$ and $\mathbf{p}_{1}=(c, s)^{\mathrm{T}}$. Let $\mathbf{q}$ be the point such that $\mathbf{q}-\mathbf{p}_{i}$ is tangent to the circle at $\mathbf{p}_{i}$ for $i=1,2$ (see Figure 10). An easy computation gives $\mathbf{q}=\left(1, \frac{1-c}{s}\right)^{\mathrm{T}}$. By using that a conic is uniquely determinated if we know two points and tangents plus a third tangent, it is sufficient to choose any line tangent to the circle at a point not belonging to the arc $\mathbf{p}_{0} \mathbf{q}_{0}$. We shall use the line $r$ with equation $y=1$ (a more symmetric choice would be the tangent line at $\mathbf{x}$ depicted in the Figure 10).

If we set

$$
\mathbf{v}_{0}=\left(\begin{array}{l}
1 \\
0 \\
1
\end{array}\right), \mathbf{v}_{1}=\left(\begin{array}{c}
s \\
1-c \\
s
\end{array}\right), \mathbf{v}_{0}=\left(\begin{array}{l}
c \\
s \\
1
\end{array}\right), \mathbf{w}=\left(\begin{array}{c}
0 \\
1 \\
-1
\end{array}\right)
$$




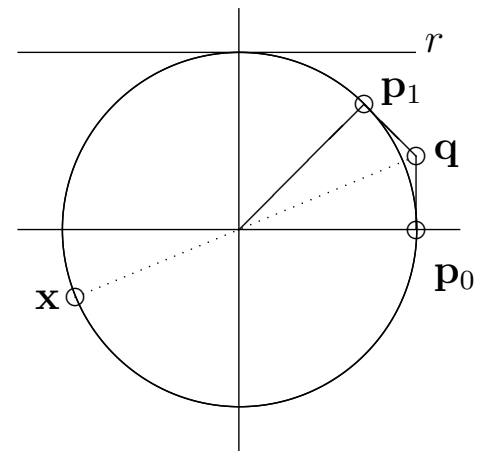

Figure 10: Construction of a circular arc.

then we can apply (10) and (11) obtaining

$$
w(t)=-(1-t)^{2}+\frac{2 t(1-t) s}{1-c-s}+\frac{t^{2}}{s-1}
$$

and

$$
\mathbf{r}(t)=\frac{1}{w(t)}\left(-(1-t)^{2}+\frac{2 t(1-t) s}{1-c-s}+\frac{c t^{2}}{s-1}, \frac{2 t(1-t)(1-c)}{1-c-s}+\frac{s t^{2}}{s-1},\right)^{\mathrm{T}} .
$$

In [6], Piegl and Tiller show that it is not possible to represent the full circle by quadratic $\mathrm{C}^{1} \mathrm{~B}$-splines. In [7] it was said that "The construction of a general NURBS circular arc is more complicated than first expected, and there are many ways to do it". Here we present a simple and intuitive method to draw a general circular arc. Moreover, by Example 3, we can establish the following result.

TheOREM 4.1 For all $\alpha \in] 0,2 \pi[$, we can construct a rational Bézier circular arc with sweep angle $\alpha$.

Example 4: Given $\mathbf{b}_{0}, \mathbf{b}_{1} \in \mathcal{E}^{2}$, draw an ellipse such that one axis is $\overline{\mathbf{b}_{0} \mathbf{b}_{1}}$ and the length of the other semiaxis is $b$ (note that a circle is a particular case).

Let $\mathbf{v} \in \mathbb{R}^{2}$ be perpendicular to $\mathbf{b}_{1}-\mathbf{b}_{0}$ with $\|\mathbf{v}\|=1$ and $\left\{\mathbf{b}_{1}-\mathbf{b}_{0}, \mathbf{v}\right\}$ a right-handed basis. Also, let $\mathbf{p}=\mathbf{b}_{0}-b \mathbf{v} \in \mathcal{E}^{2}$ and $r$ be the affine line with 
equation $(\mathbf{x}-\mathbf{p})^{\mathrm{T}} \mathbf{v}=0$ (see Figure 11$)$. Note that the equation of this line is

$$
0=\left(\mathbf{x}-\left(\mathbf{b}_{0}-b \mathbf{v}\right)\right)^{\mathrm{T}} \mathbf{v}=\mathbf{x}^{\mathrm{T}} \mathbf{v}-\mathbf{b}_{0}^{\mathrm{T}} \mathbf{v}+b=\left(\mathbf{x}^{\mathrm{T}}, 1\right)\left(\begin{array}{c}
\mathbf{v} \\
b-\mathbf{b}_{0}^{\mathrm{T}} \mathbf{v}
\end{array}\right)
$$

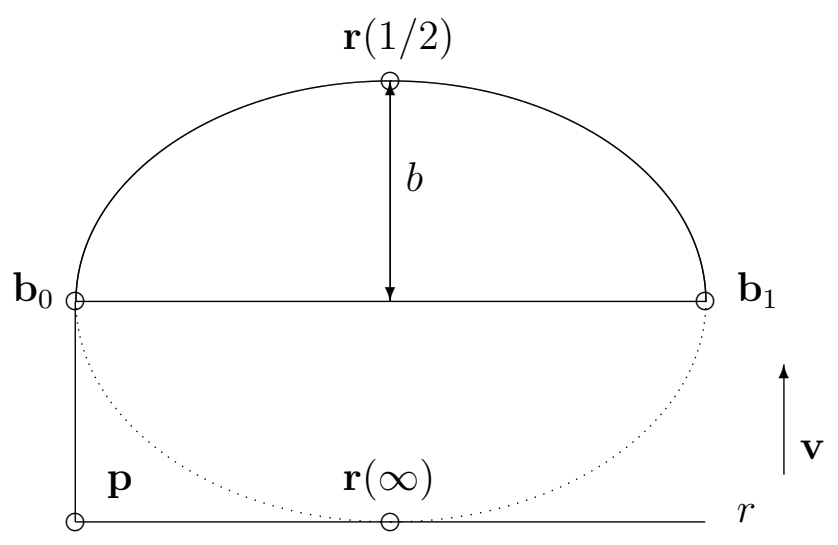

Figure 11: Construction of an ellipse.

In order to apply the Algorithm 2.1 we set

$$
\mathbf{v}_{0}=\left(\begin{array}{c}
\mathbf{b}_{0} \\
1
\end{array}\right), \quad \mathbf{v}_{1}=\left(\begin{array}{c}
\mathbf{v} \\
0
\end{array}\right), \quad \mathbf{v}_{2}=\left(\begin{array}{c}
\mathbf{b}_{1} \\
1
\end{array}\right)
$$

and the projective line $\pi^{*}(\mathbf{w})$ with $\mathbf{w}^{\mathrm{T}}=\left(\mathbf{v}^{\mathrm{T}}, b-\mathbf{b}_{0}^{\mathrm{T}} \mathbf{v}\right)$. It is easy to check $\mathbf{w}^{\mathrm{T}} \mathbf{v}_{0}=\mathbf{w}^{\mathrm{T}} \mathbf{v}_{2}=b$ and $\mathbf{w}^{\mathrm{T}} \mathbf{v}_{1}=1$. Thus, applying (10) and (11) we get

$$
w(t)=\frac{(1-t)^{2}+t^{2}}{b}
$$

and if $\mathbf{r}(t)$ is the parametrization of the ellipse, then

$$
\mathbf{r}(t)=\frac{1}{(1-t)^{2}+t^{2}}\left((1-t)^{2} \mathbf{b}_{0}+2 b t(1-t) \mathbf{v}+t^{2} \mathbf{b}_{1}\right)
$$

Notice that

$$
\mathbf{r}(1 / 2)=\frac{1}{2}\left(\mathbf{b}_{0}+\mathbf{b}_{1}\right)+b \mathbf{v}, \quad \mathbf{r}(\infty)=\frac{1}{2}\left(\mathbf{b}_{0}+\mathbf{b}_{1}\right)-b \mathbf{v},
$$

as Figure 11 shows. 
We can prove that the conic is indeed an ellipse. One way is the following: by a suitable movement, we can suppose that $\mathbf{b}_{0}=(-a, 0)^{\mathrm{T}}$ and $\mathbf{b}_{1}=(a, 0)^{\mathrm{T}}$. By applying Theorem 3.3 we obtain that the equation of this conic is $x^{2} / a^{2}+$ $y^{2} / b^{2}=1$.

What happens if $\mathbf{v}$ changes? Observe that if $\mathbf{v}$ varies, then we obtain different ellipses as the Figure 12 shows. But in this case, $b$ is not the length of one semiaxis. Notice that $\mathbf{v}$ is the direction of the tangent through $\mathbf{b}_{i}$. In fact, from (20), one easily gets $\mathbf{r}^{\prime}(0)=2 b \mathbf{v}$.

Figure 12: Construction of ellipses. Left: $\mathbf{b}_{0}=(-1,0)^{\mathrm{T}}, \mathbf{b}_{1}=(1,0)^{\mathrm{T}}$, $b=1.5$. Right: $\mathbf{b}_{0}=(-1,0)^{\mathrm{T}}, \mathbf{b}_{1}=(1,0)^{\mathrm{T}}, b=1.5, \mathbf{v}=(1,1)^{\mathrm{T}}$.

Example 5: Construct an hyperbola whose assympotes are two non parallel affine lines $r_{1}$ and $r_{2}$.

Let the equations of $r_{1}$ and $r_{2}$ be $\mathbf{x}=\mathbf{b}+\lambda \mathbf{u}_{1}$ and $\mathbf{x}=\mathbf{b}+\lambda \mathbf{u}_{2}$ respectively (we shall suppose $\left\|\mathbf{u}_{1}\right\|=\left\|\mathbf{u}_{2}\right\|=1$ ) with $\mathbf{b}=\left(x_{0}, y_{0}\right)^{\mathrm{T}}=r_{1} \cap r_{2}$ and let $r$ be another affine line with equation $a x+b y+c=0$. See Figure 13.

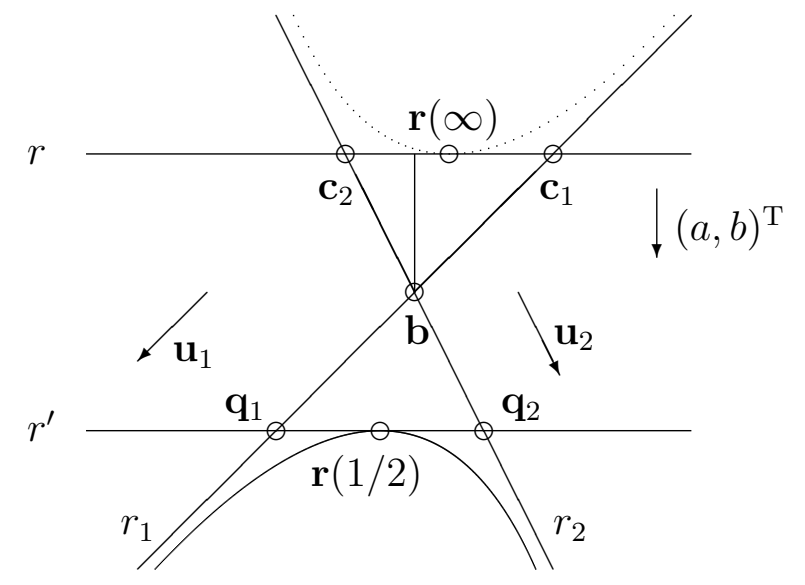

Figure 13: Construction of an hyperbola.

In order to apply the algorithm, set

$$
\mathbf{v}_{0}=\left(\begin{array}{c}
\mathbf{u}_{1} \\
0
\end{array}\right), \quad \mathbf{v}_{1}=\left(\begin{array}{c}
\mathbf{b} \\
1
\end{array}\right), \quad \mathbf{v}_{2}=\left(\begin{array}{c}
\mathbf{u}_{2} \\
0
\end{array}\right), \quad \mathbf{w}=\left(\begin{array}{c}
a \\
b \\
c
\end{array}\right)
$$


Furthermore, we require that $\mathbf{w}^{\mathrm{T}} \mathbf{v}_{i} \neq 0$ for $i=0,1,2$. These conditions are equivalent to say: a) The point $\mathbf{b}$ does not lie on the line $r$ (we shall suppose $\left.a x_{0}+b y_{0}+c>0\right)$ and b) The lines $r_{i}$ and $r$ are not parallel for $i=1,2$. From (10) we get

$$
w(t)=\sum_{i=0}^{2} \frac{B_{i}^{2}(t) z_{i}}{\mathbf{w}^{\mathrm{T}} \mathbf{v}_{i}}=\frac{2 t(1-t)}{a x_{0}+b y_{0}+c} .
$$

Let us set $k=a x_{0}+b y_{0}+c, k_{1}=\mathbf{w}^{\mathrm{T}} \mathbf{v}_{0}$, and $k_{2}=\mathbf{w}^{\mathrm{T}} \mathbf{v}_{2}$. From (11) we obtain

$$
\begin{aligned}
\mathbf{r}(t) & =\frac{1}{w(t)}\left(\frac{B_{0}^{2}(t)}{\mathbf{w}^{\mathrm{T}} \mathbf{v}_{0}} \mathbf{u}_{1}+\frac{B_{1}^{2}(t)}{\mathbf{w}^{\mathrm{T}} \mathbf{v}_{1}} \mathbf{b}+\frac{B_{2}^{2}(t)}{\mathbf{w}^{\mathrm{T}} \mathbf{v}_{2}} \mathbf{u}_{2}\right) \\
& =\frac{k}{2 t(1-t)}\left(\frac{(1-t)^{2}}{k_{1}} \mathbf{u}_{1}+\frac{2 t(1-t)}{k} \mathbf{b}+\frac{t^{2}}{k_{2}} \mathbf{u}_{2}\right) \\
& =\frac{k}{k_{1}} \frac{1-t}{2 t} \mathbf{u}_{1}+\mathbf{b}+\frac{k}{k_{2}} \frac{t}{2(1-t)} \mathbf{u}_{2} .
\end{aligned}
$$

The line $r_{1}$ is an assymptote. In fact: embedding the vectors and points in $\mathbb{R}^{3}$ and applying the following formula "the distance between the point $\mathbf{p}$ and the line $\mathbf{x}=\mathbf{b}+\lambda \mathbf{v}$ is $\|(\mathbf{p}-\mathbf{b}) \times \mathbf{v}\| /\|\mathbf{v}\|$ ", we obtain

$$
\mathrm{d}\left(\mathbf{r}(t), r_{1}\right)=\frac{\left\|(\mathbf{r}(t)-\mathbf{b}) \times \mathbf{u}_{1}\right\|}{\left\|\mathbf{u}_{1}\right\|}=\frac{k}{k_{2}} \frac{t}{2(1-t)} \frac{\left\|\mathbf{u}_{2} \times \mathbf{u}_{1}\right\|}{\left\|\mathbf{u}_{1}\right\|} \stackrel{t \rightarrow 0}{\longrightarrow} 0 .
$$

Similarly, one has $\lim _{t \rightarrow 1} \mathrm{~d}\left(\mathbf{r}(t), r_{2}\right)=0$. Hence $r_{2}$ is the other assymptote. Notice that $\mathbf{b}$ is the center of the hyperbola because $\mathbf{b}$ is the intersection of the two assymptotes.

The expression for $\mathbf{r}(t)$ can be simplified if we observe that

$$
k=\mathrm{d}(\mathbf{b}, r) \sqrt{a^{2}+b^{2}}
$$

and

$$
k_{1}=\mathbf{w}^{\mathrm{T}} \mathbf{v}_{0}=(a, b) \mathbf{u}_{1}=\sqrt{a^{2}+b^{2}}\left\|\mathbf{u}_{1}\right\| \cos \phi_{1}=\sqrt{a^{2}+b^{2}} \cos \phi_{1},
$$

where $\phi_{1}$ is the angle between $(a, b)^{\mathrm{T}}$ and $\mathbf{u}_{1}$ (see Figure 13). Analogously, denoting by $\phi_{2}$ the angle between $(a, b)^{\mathrm{T}}$ and $\mathbf{u}_{2}$, we get $k_{2}=\sqrt{a^{2}+b^{2}} \cos \phi_{2}$. Hence

$$
\frac{k}{k_{i}}=\frac{\mathrm{d}(\mathbf{b}, r)}{\cos \phi_{i}}=\mathrm{d}\left(\mathbf{c}_{i}, \mathbf{b}\right)
$$


being $\mathbf{c}_{i}$ the intersection of $r$ and $r_{i}$. Thus,

$$
\mathbf{r}(t)=\mathrm{d}\left(\mathbf{c}_{1}, \mathbf{b}\right) \frac{1-t}{2 t} \mathbf{u}_{1}+\mathbf{b}+\mathrm{d}\left(\mathbf{c}_{2}, \mathbf{b}\right) \frac{t}{2(1-t)} \mathbf{u}_{2} .
$$

Observe that

$$
\mathbf{r}(\infty)=-\frac{1}{2}\left(\mathrm{~d}\left(\mathbf{c}_{1}, \mathbf{b}\right) \mathbf{u}_{1}+\mathrm{d}\left(\mathbf{c}_{2}, \mathbf{b}\right) \mathbf{u}_{2}\right)+\mathbf{b}
$$

and taking into account that $\mathbf{b}-\mathbf{c}_{i}=\mathrm{d}\left(\mathbf{c}_{i}, \mathbf{b}\right) \mathbf{u}_{i}$, we get

$$
\mathbf{r}(\infty)=\frac{\mathbf{c}_{1}+\mathbf{c}_{2}}{2}
$$

as Figure 13 shows. Also, notice that the shoulder point is

$$
\mathbf{r}(1 / 2)=\frac{1}{2}\left(\mathrm{~d}\left(\mathbf{c}_{1}, \mathbf{b}\right) \mathbf{u}_{1}+\mathrm{d}\left(\mathbf{c}_{2}, \mathbf{b}\right) \mathbf{u}_{2}\right)+\mathbf{b} .
$$

The geometric interpretation of the shoulder point is the following: Let $\mathbf{q}_{i}$ be the point such that $\mathbf{b}$ is the midpoint of $\overline{\mathbf{q}_{i} \mathbf{c}_{i}}$ (see again Figure 13). Recalling that $\mathbf{b}-\mathbf{c}_{i}=\mathrm{d}\left(\mathbf{c}_{i}, \mathbf{b}\right) \mathbf{u}_{i}$ and $\mathbf{b}=\left(\mathbf{c}_{i}+\mathbf{q}_{i}\right) / 2$, we get

$$
\mathbf{r}(1 / 2)=\frac{\mathbf{q}_{1}+\mathbf{q}_{2}}{2}
$$

Moreover, if $r^{\prime}$ is the symmetric line to $r$ respect to $\mathbf{b}$, we obtain that $r^{\prime}$ is tangent to the hyperbola at the shoulder point. This latter fact, again, shows the importance of the choice of the auxiliary line $r$ in the input of the Algorithm 2.1.

\section{References}

[1] W. Boehm, H. Prautzsch. Geometric concepts for geometric design. A.K. Peters, (1994).

[2] D.A. Brannan, M.F. Esplen, J.J. Gray. Geometry. Cambridge University Press, (1999).

[3] G. Farin. Algorithms for Rational Bézier Curves, C.A.D., Vol 15, No 4, pp 73-77, (1983). 
[4] G. Farin. Curves and surfaces for computer aided geometric design. Academic Press (1997).

[5] M. Penna, R. Patterson. Projective geometry and its applications to computer graphics. Prentice Hall, (1986).

[6] L. Piegl, W. Tiller. A menagerie of rational B-spline circles, IEEE Comput. Graph. and Appl. Vol. 21. No. 9, pp 48-56, (1989).

[7] L. Piegl, W. Tiller. The NURBS book. Springer, (1997). 